\section{Men with ED have an increased risk of developing Parkinson's disease}

Erectile dysfunction (ED) is common in men with Parkinson's disease, perhaps because both disorders are associated with dopamine and testosterone status. Gao and colleagues' analysis of retrospective data from the Health Professionals Study has now shown that ED might precede the onset of Parkinson's motor symptoms by many years, and might help to identify men with an increased risk of developing Parkinson's disease.

In total, 32,616 Health Professionals Study participants without Parkinson's disease at baseline in 1986 responded to a questionnaire, mailed in 2000, that assessed their erectile function between 1984 and 2000. Compared with men who reported very good erectile function before 1986, men who reported first onset of ED before 1986 were four times more likely to develop Parkinson's disease by 2002 $(P<0.0001)$. After adjustment for potentially confounding variables, the prevalence of ED in the 200 men who had developed Parkinson's disease by 2000 was much higher than in men who had not (68\% versus $32 \%)$. This association was evident in all age-groups, but was strongest in men with the youngest age at onset of ED.

Gao and colleagues note that ED and constipation (another common complaint in men who eventually develop Parkinson's disease) are both manifestations of parasympathetic cholinergic failure. They conclude that symptoms of autonomic dysfunction might be evident years before Parkinson's disease is clinically recognizable.

Original article Gao X et al. (2007) Erectile function and risk of Parkinson's disease. Am J Epidemiol 166: 1446-1450

\section{Successful use of the appendix for ureteral replacement: report of 10 pediatric cases}

Ureteral replacement is rarely necessary in children, and various surgical procedures have been described for this purpose. One such procedure involves the use of the appendix as a ureteral substitute; however, only isolated reports of this approach exist in the literature. In their paper published in the Journal of Pediatric Urology, Dagash and colleagues have documented the largest series to date of patients treated with this approach.

The authors reviewed case notes on 10 children from three centers in the UK and India. Mean age at surgery was 2.5 years (range 2.5 months to 12 years). The appendix was substituted for the right ureter in nine patients, and for the left ureter in the remaining child. In all patients, the appendix was positioned in an antiperistaltic orientation and anastomosed to the renal pelvis via a cecal cuff. The underlying conditions necessitating ureteral replacement were congenital ureteral stenosis $(n=5)$, nondrainage following pyeloplasty $(n=3)$, traumatic ureteral avulsion $(n=1)$ and stricture following ureteral reimplantation for vesicoureteral reflux $(n=1)$. Median duration of follow-up was 16 months (range 1-72 months).

Following surgery, renal function was preserved in nine children. In the remaining child, a 2-year-old boy with right ureteral stricture and only $36 \%$ preoperative right differential renal function, the right kidney was nonfunctional at 8 months. The authors conclude that the appendix can safely and effectively be used as a ureteral substitute in children, even very young babies.

Original article Dagash $\mathrm{H}$ et al. (2008) The appendix as ureteral substitute: a report of 10 cases. J Pediatr Urol 4: 14-19

\section{A low PSA level does not always indicate favorable prognosis}

Serum PSA levels are used to predict the prognosis of patients with prostate cancer, but assessment of the prognostic accuracy of PSA values is difficult, particularly in populations in which screening programs probably confound the data. Sandblom et al. assessed the prognostic value of PSA levels in a large cohort of men with prostate cancer in Sweden, a country without a screening program and with a conservative approach to the management of prostate cancer.

From a national cancer register, the researchers identified 28,531 men aged $<75$ years with prostate cancer characteristics indicative of curable disease (i.e. tumor localized to the prostate and a PSA level $<20 \mathrm{ng} / \mathrm{ml}$ ). Sandblom and colleagues estimated the 